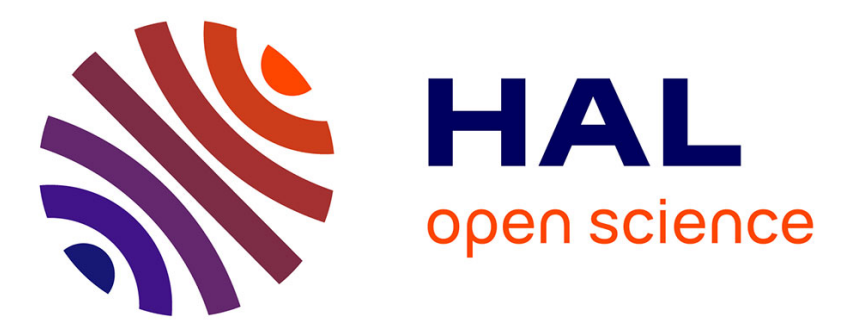

\title{
TRIZ Barcamp Exercise: Ideas for Wearable Defibrillators
}

Tiziana Bertoncelli, Barbara Gronauer, Horst Nähler

\section{To cite this version:}

Tiziana Bertoncelli, Barbara Gronauer, Horst Nähler. TRIZ Barcamp Exercise: Ideas for Wearable Defibrillators. 19th International TRIZ Future Conference (TFC), Oct 2019, Marrakesh, Morocco. pp.257-265, 10.1007/978-3-030-32497-1_21. hal-02905556

\section{HAL Id: hal-02905556 \\ https://hal.inria.fr/hal-02905556}

Submitted on 23 Jul 2020

HAL is a multi-disciplinary open access archive for the deposit and dissemination of scientific research documents, whether they are published or not. The documents may come from teaching and research institutions in France or abroad, or from public or private research centers.
L'archive ouverte pluridisciplinaire HAL, est destinée au dépôt et à la diffusion de documents scientifiques de niveau recherche, publiés ou non, émanant des établissements d'enseignement et de recherche français ou étrangers, des laboratoires publics ou privés.

\section{(c)(1)}

Distributed under a Creative Commons Attribution| 4.0 International License 


\title{
TRIZ Barcamp Exercise: Ideas for Wearable Defibrilla- tors
}

\author{
Tiziana Bertoncelli ${ }^{1}$, Barbara Gronauer ${ }^{2}$, Horst Nähler ${ }^{3}$ \\ ${ }^{1}$ TRIZ Consulting Group, Sulzbach-Rosenberg, Germany \\ ${ }^{2}$ StrategieInnovation, Hünfeld, Germany \\ ${ }^{3}$ c4pi - Center for Product-Innovation, Hünfeld, Germany \\ bertoncelli@triz-consulting.de
}

\begin{abstract}
A wearable external defibrillator is usually an option for all patients at risk for sudden cardiac arrest (SCA) for whom the very invasive surgery intervention to implant an internal defibrillator is not viable. The metallic paddles that convey the electrical discharge have to stay attached to the skin for effectiveness of the device, but such a solution presents several problems, i.e. lack of comfort, difficulties in usage for overweight people, skin rash and burns, itching, sleep disturbances. Moreover, the vest be removed to shower or bathe. As a result, many patients stop using it, putting themselves at a very high risk. Such a system presents a lot of issues and contradictions and an effective use of TRIZ methodology could be very helpful to identify the needed improvement actions. The present paper will show a case study model developed for the first TRIZ Barcamp, with strong focus on the modelling peculiarities required by a very short session, the ideas generated by the participants and the learnt lessons.
\end{abstract}

Keywords: Wearable Defibrillator, Barcamp, Contradictions, Functional Analysis.

\section{A TRIZ Session for a wearable defibrillator}

\subsection{The TRIZ Barcamp experience}

The present paper will show a case study model developed for the first TRIZ Barcamp, focusing on the modelling peculiarities required by a very short session; also the ideas generated by the participants and the learnt lessons will be discussed. The first TRIZ Barcamp took place in Fulda, Germany, in Februar 2018, following the international format spread by the Barcamp International Network [1]. Originated in the Information Technology field, especially for web application, this unconference concept spread very fast to many other fields.

The content of such an open workshop is chosen at the beginning of the session by the participants themselves.

During the TRIZ Barcamp, attended by $\sim 25$ people, different topics were presented at the very beginning, presented as problems to be tackled by means of TRIZ; every short oral presentation was actiually a call for participants, where the number of people, the level of needed TRIZ expertise and the approximated required time. After the brief 
topic introductions, the participants declared their interest and willingness to attend the dedicated session (about 20 minute each), and both the content and the agenda of the workshop were organized accordingly.

\subsection{The Wearable defibrillator session}

The wearable defibrillator topic was proposed and selected by the Barcamp participants for a 20-minute session, for which all level of TRIZ expertise were welcome. It was introduced explaining which kind of patient need such a device and the issues it poses, as explained in detail in Paragraph 2. Since a very short time was allocated for the Barcamp exercise, the TRIZ modelling phase had already been sketched during a prework session and presented to the participants. The proposed TRIZ tools to be dynamically selected for the problem-solving tools were Physical Contradictions and Substance-Field modeling. A Function Model was also prepared, to serve as an example and guideline. Goal of the workshop was to identify solutions as effective as the implanted defibrillator, but with a lesser degree of invasiveness; the workshop was kicked off posing no boundary to the kind of ideas and with the statement that ideas were to the be released and published afterwards.

\section{Wearable defibrillator: importance and system description}

The wearable external defibrillator is regarded as a valuable option for all patients at risk for sudden cardiac arrest (SCA). Many cardiovascular conditions require continuous monitoring and the possible intervention with a defibrillator, i. e. recent myocardial infarctions, inherited arrythmias, and congenital heart diseases [2-6]. The usual way to prevent a sudden arrhythmia in a patient is to implant an internal defibrillator, with a very invasive surgery intervention. When surgery is not possible or before it, the wearable defibrillator offers an alternative. This solution nevertheless poses issues that impair its long-term usage and effectiveness. A substantial improvement of such a system would be of major impact also for patients with limited access to healthcare for geographic and economic reasons, i.e. the ones living in rural areas, and the ones for whom surgery is an economical challenge.

The system typically [2-6] comprises a lightweight vest, a monitoring system and the necessary electrodes to perform the monitoring function and to convey the electrical discharge when an anomaly is detected, as shown in Fig 1.

The usual reported problems are:

- The person wears the defibrillator all day, but it must be removed to shower or bathe, exposing the patient at risk

- The electrodes need to press against the chest firmly enough to detect heart rhythm changes, but not so tightly that they trigger false alarms

- The defibrillator vest is not comfortable 
- It is difficult to use if person is overweight

- Up to one in five people stop using it because of: skin rash, itching, sleep disturbances, skin burns

These issues represented the starting point of the TRIZ modelling effort, explained in Paragraph 3.
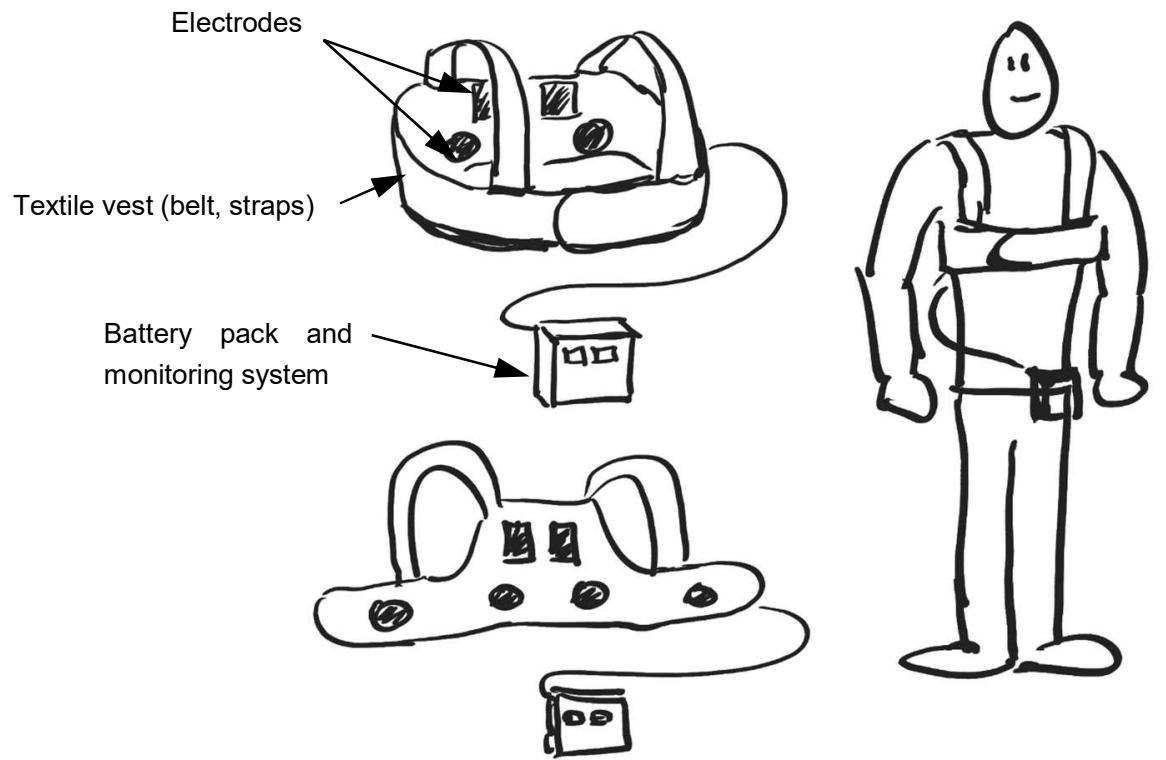

Fig. 1. Sketch of a typical wearable defibrillator.

\section{Wearable defibrillator: main aspects and TRIZ modelling}

In addition to the well-known disadvantages listed in Paragraph 2, the main aspect that can be expressed as an Engineering Problem is the following: the metallic paddles needed to convey the electrical discharge have to stay attached to the skin for effectiveness of the devices; nevertheless such a solution is not practical since the metallic material damages the skin in the long run. This disadvantage has a very localized operating zone and depicts a harmful interaction; it can be thus very effectively modeled by means of Physical contradictions and a Su-Field Models, as shown in details in Paragraphs 3.1.and 3.2. Those models were prepared as a pre-work prior to the Barcamp day; the 20-minute session would have not allowed both a modelling and a problem solving stage, so it was preferred to involve the participants 
in the latter only; the two chosen modeling approaches, being the Su-Field model visual and the physical contradictions focused on the core physics of the problem have been also proven to be the most effective for very short sessions, especially when the participants have little or no previous TRIZ experience [7].

\subsection{Physical Contradictions}

The physical contradiction presented to the session participants was formulated as follows:

Electrodes SHOULD BE attached to the skin

to monitor heart and convey electrical discharge

Electrodes SHOULD NOT BE attached to the skin not to irritate, damage or burn it

The workshop participants immediately observed that the approach Separation in Space was not an option, being the electrode placement fixed; nevertheless they recognized quickly that the requirements to fulfill could be expressed as two functions: Monitoring and Convey Electrical Discharge, to be delivered at two different times; a more precise modelling would have demanded to address the two operating modes separately. For this reason, the physical contradiction was refined and split accordingly:

Monitoring Function

Electrodes SHOULD BE attached to the skin

to monitor heart

Electrodes SHOULD NOT BE attached to the skin

not to irritate or damage it

Restart Heartbeat Function

Electrodes SHOULD BE attached to the skin

to convey electrical discharge

Electrodes SHOULD NOT BE attached to the skin

not to irritate, damage or burn it

\subsection{Substance-Field Model}

The most acute harmful interaction occurs between the defibrillator paddles (electrodes) and the skin; during monitoring because of the mechanical friction and during the electrical discharge because of the electric current and the associated thermal field, as summarized in Fig. 2: 


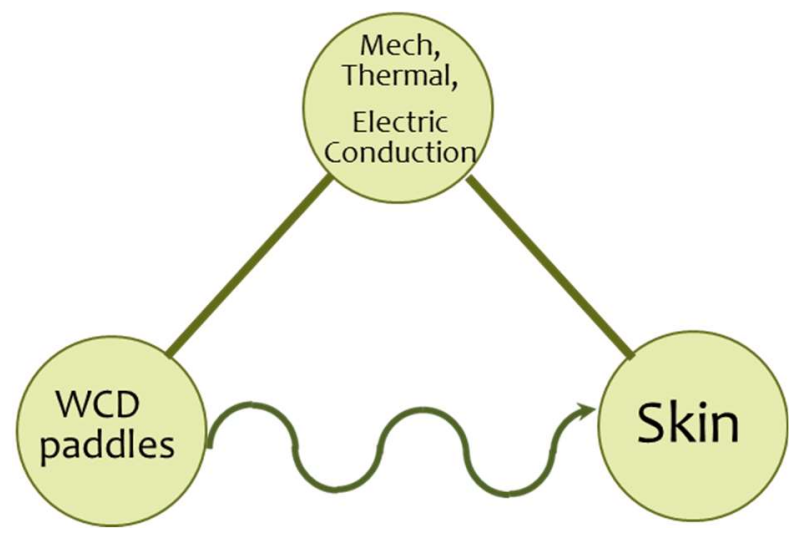

Fig. 2. Substance-field model for a wearable cardioverter defibrillator (WCD): the electrode paddles generate mechanical friction, electric conduction and heat, damaging the skin (harmful interaction).

\subsection{Functional Analysis}

This function model was briefly presented to the participants, mostly for illustrating purposes, to showcase this approach to the newbies and as a back-up for future inspiration. It reported in Fig. 3, where the System component are depicted in green, the Supersystem ones in blue; the Target of the main function is highlighted in cyan; it is composed of heart muscles, heart electric field and blood for simplicity of modelling. 


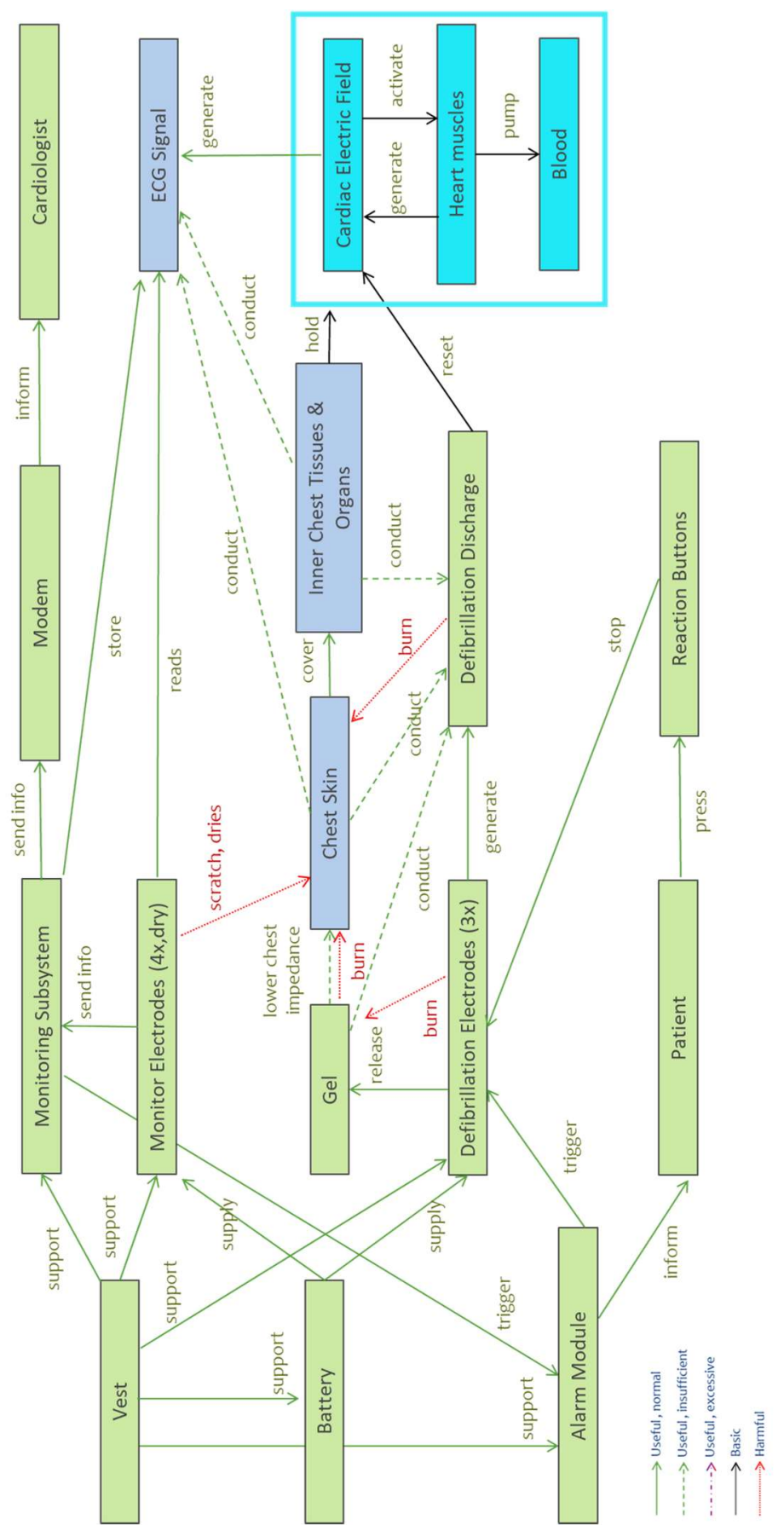

Fig. 3. Function model for a wearable defibrillator 


\section{Idea List Description, Discussion, Open Points}

Many ideas ( 20) were generated (Fig 4) and subsequently grouped according to the kind of proposed intervention.

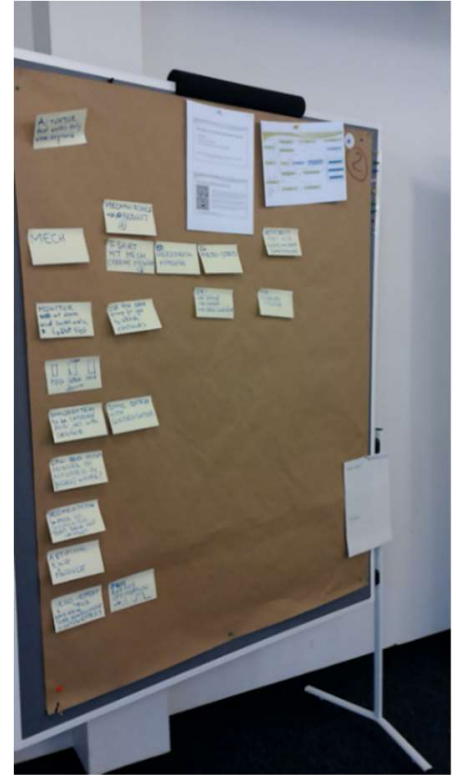

Fig. 4. Generated Ideas

\section{Modify Vest}

1. Use an actuator that compresses shock electrodes on the skin only when arrhythmia detected, using same mechanism (pump) that pushes conductive gel to attach electrodes

2. Use segmented pads: single pad surface so small that skin not damaged, with many small contact surfaces, like lotus-effect or gecko-foot

\section{Modify Monitoring}

1. Monitor also through blood pressure

2. Monitor by means of a smartwatch-like device (recognized in a commercial product in November 2018)

\section{Minimize battery size for low weight Vest}

1. Use smaller battery + condensator 
2. Make it chargeable with net current, auto with a sensor signaling when recharge is needed

3. Use piezo-actuators with body movements

\section{Use other fields (not electrical)}

1. T-Shirt with monitoring + mechanical cardiac massage

2. Use piezo-strips for the abovementioned cardiac massage

3. Restart heartbeat not with an electrical shock, but with acoustic field

4. Restart heartbeat not with an electrical shock, but mechanically

5. Activate heart muscles with other impulses (e.g. microwaves?)

6. Use a mixed approach, implementing a smaller shock + mechanical cardiac massage

\section{Act on ECG}

1. Produce an artificial ECG

2. Study energy optimization of the needed electric pulse: amplitude \& duration

Use another substance S3* between pads and skin to improve conductivity - Modified T-shirt

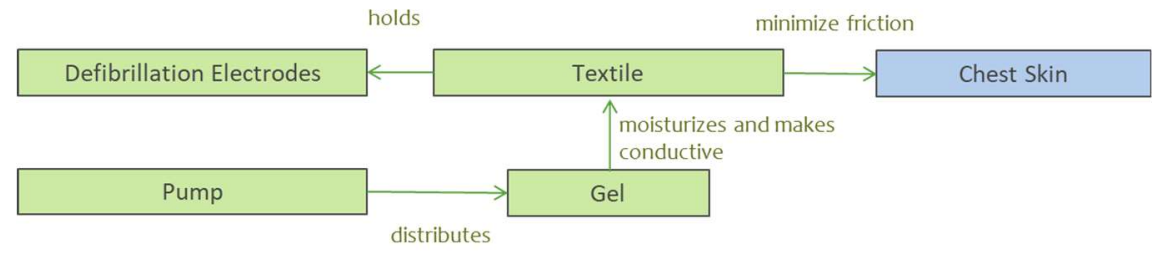

Fig. 6. Function Model of a solution

1. Just under the electrodes use a patch of special textile that gets conductive when wet conductive gel is pumped on it

2. Use a mixed textile: e. g. combination of cotton + metal

Use another substance $\mathrm{S3}$ * between pads and skin to improve conductivity use available body fluids

1. Blood

2. Sweat

3. Skin Moisture 


\section{Conclusions: lessons learnt}

The conclusions can be grouped in two categories: First the immediate observations and the feedback of the participating group, second the observations from the external viewpoint of the TRIZ-facilitator.

Conclusions from immediate observation and feedback:

- The number of ideas and the engagement of the participants confirmed the effectiveness of visual methods like Su-Field models and Physical contradictions

- $\quad$ TRIZ-initiated participants appreciated the pre-prepared modelling effort and enjoyed the creative process

- Non-TRIZ experts were intimidated by the TRIZ-jargon and expressed their wish for more background information about the topic, that resonated quickly but the impact of which needed to be better clarified

- During the idea generation process the time was too short to address more technical questions, i.e.: how much energy is needed for a defibrillator discharge, which is the maximum time window for defibrillator intervention and how many people are affected.

Conclusions from external observations from the viewpoint of TRIZfacilitator/moderator:

- For short TRIZ sessions it is crucial to have a thoroughly prepared problem analysis to point out the crucial key problems to be addressed during the session. The Innovation Situation Questionnaire [8] could be used for preparation. The goal of the session and the desired solution space need also to be defined.

- The use of graphical representations is extremely helpful for explaining context and correlations regarding the system structure (TRIZ Function Model) or the problem situation (Substance Field Model, Contradictions). These tools, when used with common language, are also understandable for the "TRIZ-newcomer".

- The language and syntax used in the TRIZ Function Model helps to quickly understand the system structure and interactions, enabling participants from all different kinds of knowledge background to collectively focus on the task at hand.

- The TRIZ solution models (e.g. Standard Solutions, Inventive Principles, Separation Principles) need explanation or even re-wording by the moderator, ideally also prepared in advance. Examples for the problem models help understand the underlying meaning of the solution model and help overcoming the psychological inertia and look in different directions 
offered by TRIZ. Unmodified use of e.g. the standard solutions is likely to alienate the TRIZ newbie, making it harder to focus on idea generation because it takes time to figure out what the solution model is about.

- The role of a moderator is mandatory, especially for short, focused TRIZ sessions. It is compulsory that the process of the TRIZ session is monitored as well as the discussions between the participants. Especially short TRIZ sessions need a "game plan" and a structure beforehand, balancing time for analysis, discussion, free association and guided idea generation with TRIZ tools. Discussions between the participants need to be monitored and limited if necessary, to stay on track. If TRIZ newbies are participating, it is even more needed to have a "translator" for the TRIZ-specific syntax and wording.

The generated ideas were encouraging and it is planned to look for practical implementation of the most promising concepts.

\section{Acknowledgments}

The authors would like to thank Ms. Paola Mainardi for passionately raising the topic during a TRIZ discussion and Dr. Raffaella Boise for her engaging lecture about cardiovascular diseases.

\section{References}

1. https://en.wikipedia.org/wiki/BarCamp

2. http://lifevest.zoll.com/de/medical-professionals/

3. https://en.wikipedia.org/wiki/Wearable_cardioverter_defibrillator

4. Francis, J., Reek, S. "Wearable cardioverter defibrillator: A life vest till the life boat (ICD) arrives”, Indian Heart J. 2014 Jan-Feb; 66(1): 68-72. Published online 2014 Jan 8. doi: 10.1016/j.ihj.2013.12.050

5. Wäßnig, N., "Experience With the Wearable Cardioverter-Defibrillator in Patients at High Risk for Sudden Cardiac Death", Circulation. 2016 Aug 30; 134(9): 635-643. Published online 2016 Aug 29. doi: 10.1161/CIRCULATIONAHA.115.01912

6. https://www.hrsonline.org/content/download/18186/804164/file/Wearable-CardioverterDefibrillator.pdf

7. Bertoncelli T., Mayer O., Lynass M., “Creativity, Learning Techniques and TRIZ”, ETRIA TRIZ Future Conference 2015, Berlin, October 26-29 2015

8. Terninko J., Zusman A., Zlotin B., „Systematic Innovation: An Introduction to TRIZ (Theory of Inventive Problem Solving) “, CRC Press, 1998 